\title{
Application of shape optimization method to artificial leaf design
}

\author{
M. Shimoda ${ }^{1} \&$ Y. Nakata ${ }^{2}$ \\ ${ }^{1}$ Department of Advanced Science and Technology, \\ Toyota Technological Institute, Japan \\ ${ }^{2}$ Toshiba Corporation, Japan
}

\begin{abstract}
It is considered that the shapes of plant leaves are optimized under various environmental constraints. In this paper, the optimality of a leaf shape is examined by using a numerical shape optimization method. For leaves, it is necessary to have a sufficient surface area for receiving sunlight while also being stiff enough to maintain their shapes against external loads such as gravity and winds. This study focuses on an evergreen leaf with a V-shaped cross-section and a thick blade for designing the optimal shape of an artificial leaf. Stiffness, amount of sunlight received and volume are used as the response functions to evaluate leaf performance. A node-based non-parametric method with pyramidshaped basis vectors is used as the shape optimization method. The trade-off problem between the amount of sunlight received and stiffness is solved with this method. Prior to leaf shape optimization, calculated examples in engineering structural design are presented to verify the effectiveness of this method. The leaf shape optimization results show that the optimal V-shaped cross-sections were obtained according to the sunlight direction and target stiffness.

Keywords: artificial leaf, shape optimization, pyramid-shaped basis vector, stiffness, sunlight, V-shaped cross section.
\end{abstract}

\section{Introduction}

Plants have evolved by adapting to environmental stimuli. The shapes and functions of their roots, stems, branches, leaves and other organs have been determined in the process. Many researchers have been interested in this adaptive growth, and have gained numerous insights into plant shapes [1-6]. In this work, 
we focused on the leaf shape and examined the optimal shape of the leaf blade for designing artificial leaves. Figure 1 shows a sketch of a plant leaf. The leaf consists of a petiole and a blade with main and lateral veins. The thin net-like veins running in the blade serve to transport nutrients. From a structural point of view, the leaf is a cantilever beam under a downward distributed load. The petiole connects the blade to the stem, which has high bending rigidity for supporting the blade against gravity and low torsional rigidity for twisting to reduce wind drag on the blade. The relations between the cross-sectional shape and various properties of the petiole are investigated in reference [7]. The blade has an organ that photosynthesizes organic compounds in response to light. The best shape for receiving the most light is one where the leaf blade is extended perpendicular to the direction of the sunlight. Leaves require sufficient stiffness and strength to keep their form exposed to the sun despite the effects of gravity and winds. A trade-off exists here. Figure 2 shows photos of a summer green leaf (elm) and an evergreen leaf (camellia). The blades of summer green plants are generally thin and have thick veins. In contrast, evergreen leaves are thick and have V-shaped cross-sections. Figure 3 shows a typical V-shaped leaf of a rubber tree.

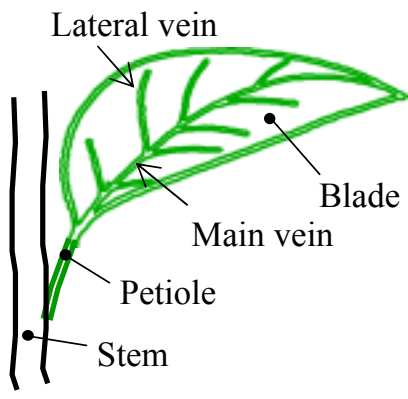

Figure 1: $\quad$ Sketch of a leaf.
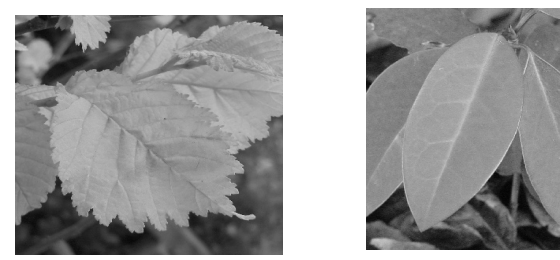

Figure 2: $\quad$ Photos of a summer green leaf (left), an evergreen leaf (right).

In this work, we focus on an evergreen leaf for designing the optimal shape of an artificial leaf, not for designing the topology of the veins. Stiffness, amount of sunlight received and volume are used as the response functions, and a simplified model of the leaf discretized by bar and shell elements is used for evaluation by finite element analysis. In the field of mechanical or structural engineering, 


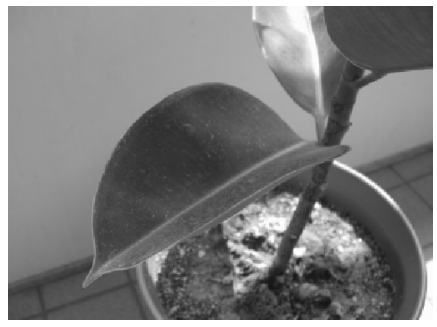

Figure 3: $\quad$ Photo of typical V-shaped rubber leaf.

numerical shape optimization techniques are often used to design optimal shapes. In this work, we applied a shape optimization method to the leaf blade in order to find its optimal shape and to solve the trade-off problem mentioned above. A node-based method with pyramid-shaped basis vectors is used, which yields the optimal shape without any shape parameterization.

The method is described in the following section along with calculated examples of basic engineering structural design problems. After that, the method is applied to leaf shape optimization problems.

\section{Shape optimization method}

\subsection{Optimization problem}

A general optimization problem can be mathematically formulated as follows:

$$
\begin{gathered}
\text { Minimize } f(\boldsymbol{x}) \\
\text { subject to } \quad g_{i}(\boldsymbol{x}) \leq \hat{g}_{i}, \quad i=1, \cdots, M \\
x_{j}^{L} \leq x_{j} \leq x_{j}^{U}, \quad j=1, \cdots, N,
\end{gathered}
$$

where $f(\boldsymbol{x})$ is the objective function. $g_{i}(\boldsymbol{x})$ and $\hat{g}_{i}$ are the $i$ th behavioral constraint function and its constraint value, respectively. $x_{j}, x_{j}^{L}$ and $x_{j}^{U}$ are the $j$ th design variable and its lower and upper side constraints, respectively. $M$ and $N$ are the total numbers of the constraints and the design variables. $x_{j}, f(\boldsymbol{x})$ and $g_{i}(\boldsymbol{x})$ for leaf shape optimization are explained in the following section.

This optimization problem can be solved by using a mathematical programming. The feasible direction method was used in this work.

\subsection{Pyramid-shaped basis vector method}

In a shape optimization problem, shape parameterization is generally needed and is an important process for defining shape perturbations, but it requires considerable experience. The obtained optimal shape is strongly influenced by parameterization. The basis vector method, one of the shape optimization methods, is used in this work. Shape updating in the basis vector method is defined as a linear combination of pre-defined basis vectors of shape perturbations as follows: 


$$
\boldsymbol{G}(\boldsymbol{x})=\boldsymbol{G}_{0}+\sum_{i=1}^{K} x_{i} \boldsymbol{T}_{i},
$$

where $\boldsymbol{G}(\boldsymbol{x})$ is the vector of nodal coordinates, $\boldsymbol{G}_{0}$ is the vector coordinates of the initial shape, $\boldsymbol{T}_{i}$ is the $i$ th basis vectors (grid perturbation vector), and $K$ is the total number of shape design variables $x_{i}$.

In order to avoid the difficulties inherent in parameterization, a general basis vector method with global basis vectors as shown in Fig. 4 is first modified. This is done by using node-based or node-by-node pyramid-shaped basis vectors like the shape functions in the finite element method (FEM) to define the shape variations as shown in Fig. 5(a). Pyramid-shaped basis vectors with unit magnitude are set for all nodes in the normal direction to the surface. Figure 5(b) shows other pyramid-shaped basis vectors with grouped elements, which is based on the concept of the design element method [8] to avoid the problem of jagged boundaries.

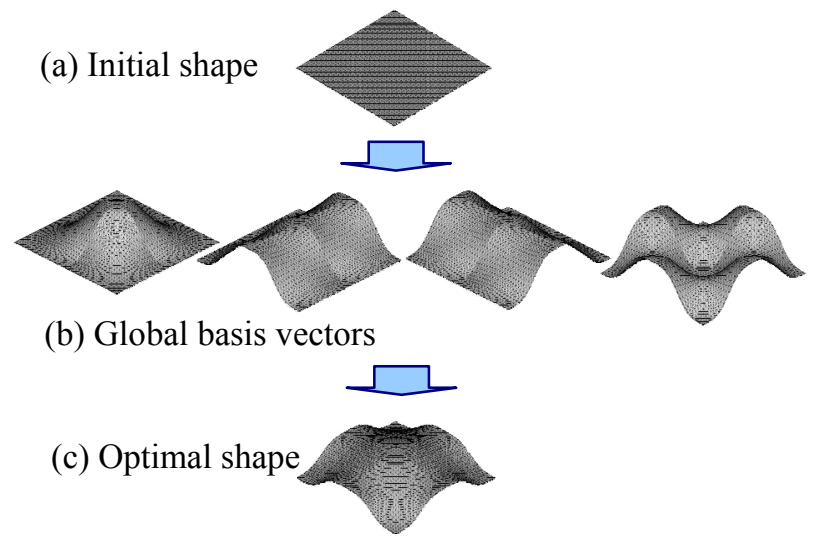

Figure 4: Example of global basis vectors.

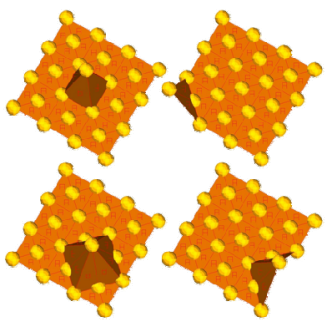

(a) Node by node

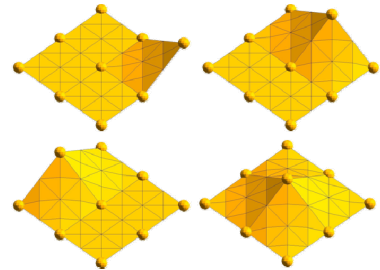

(b) Grouped

Figure 5: $\quad$ Examples of pyramid-shaped basis vectors. 


\subsection{Sensitivity analysis}

Since the optimization algorithm uses gradient information to determine the search direction, it is necessary to calculate the derivatives of the response functions, i.e., objective and constraint functions. Discrete sensitivity is calculated by FEM. In the pyramid-shaped basis vector method, the number of design variables is much larger than the number of response functions, so the adjoint method is more efficient than the direct method [9].

In the adjoint method, the derivative of the stiffness matrix $\boldsymbol{K}$ with respect to the design variables $x_{i}$ is needed. A semi-analytical method is used to evaluate it directly by using central finite differences as follows:

$$
\frac{\partial \boldsymbol{K}}{\partial x_{i}}=\frac{\partial \boldsymbol{K}\left(x_{i}+\Delta x_{i}\right)-\partial \boldsymbol{K}\left(x_{i}-\Delta x_{i}\right)}{2 \Delta x_{i}} .
$$

\subsection{Calculated examples of structural designs}

In order to verify the effectiveness of the shape optimization method with nodebased pyramid-shaped basis vectors, two calculated examples of engineering structural designs are presented. Node-based vectors were fixed through the optimization process in all calculations.

\subsubsection{Plate under torsion}

A simple stiffness design problem of a square plate under torsion was solved in one example. After defining the initial, side constraint and move limit values of the design variables, the compliance was minimized subject to a volume constraint. The initial shape and the boundary condition for this problem are shown in Fig. 6(a). The area constraint value was defined as $105 \%$ of the initial shape. Figure 6(b) shows the optimal shape obtained. The result shows that an ideal X-type bead was created for stiffening the plate. The compliance was minimized by about $96 \%$ of the initial shape, while satisfying the volume constraint.

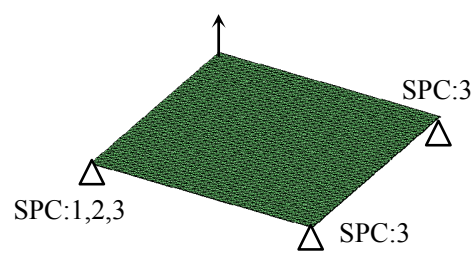

(a) Initial shape under torsion

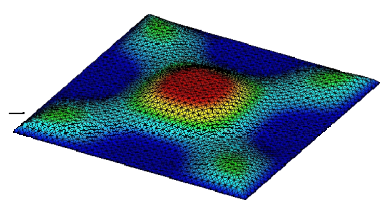

(b) Obtained shape

Figure 6: Optimization example in structural design (plate under torsion).

A roof under a snow load, i.e., a downward distributed force, was optimized in the second example. The initial shape and the boundary condition for this problem are shown in Fig. 7(a). After defining the initial and side constraints of 


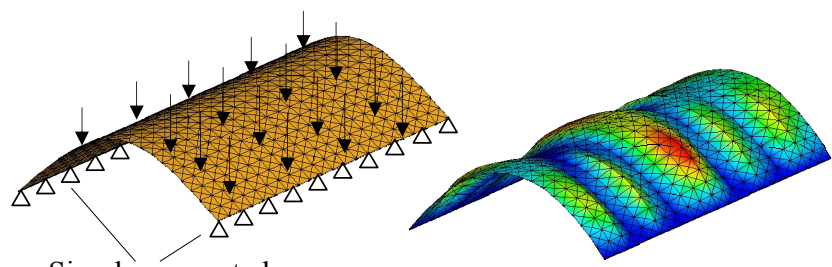

Simply supported

(a) Initial shape and boundary condition

(b) Obtained shape

Figure 7: Optimization example in structural design (roof under snow load with multi-constraints).

the design variables, the volume was minimized subject to four behavioural constraints, i.e., buckling load $(\geq 4$ times the initial), compliance ( $\leq 20 \%$ of the initial ), max. von Mises stress ( $\leq 40 \%$ of the initial ) and the first natural frequency $(\geq 1.5$ times the initial $)$. Figure $7(b)$ shows the optimal shape obtained. The result shows that several beads were created along the circumferential direction. Iteration convergence histories of the response functions are shown in Fig. 8. The values were normalized to those of the initial shape. The volume was increased by $6 \%$ over the initial shape because of the strict constraints, while satisfying the given constraints. Figure 9 shows the shape convergence histories from the initial shape (a) to the final shape (h).

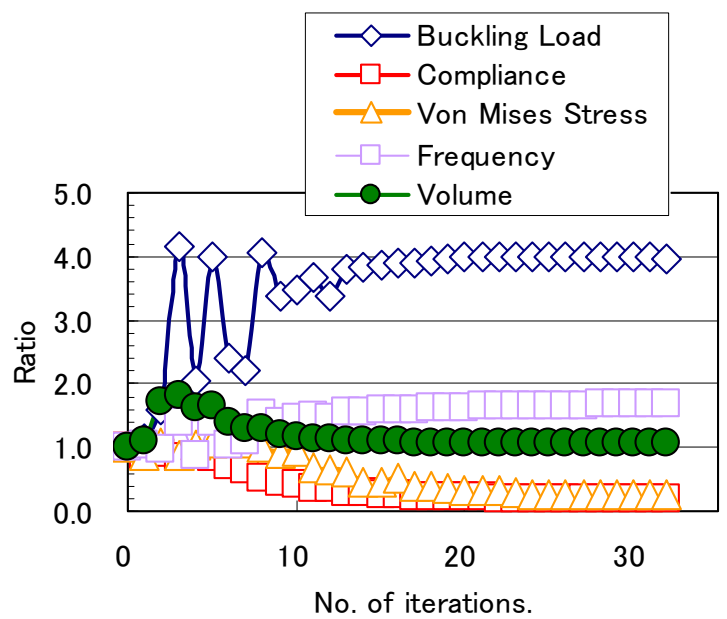

Figure 8: Iteration histories in roof optimization example. 

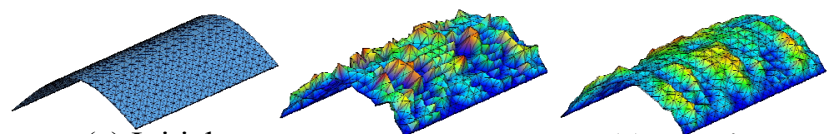

(a) Initial

(b) Iteration 5

(c) Iteration 10
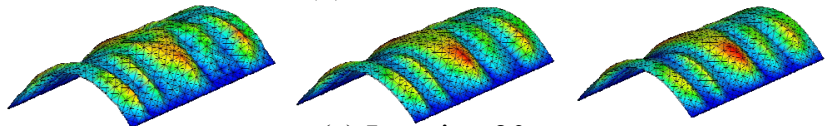

(d) Iteration 15

(e) Iteration 20

(f) Iteration 25

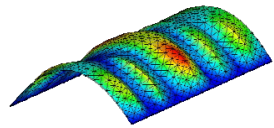

(g) Iteration 30

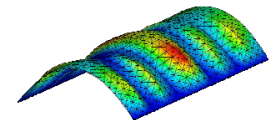

(h) Final

Figure 9: $\quad$ Shape histories in roof optimization example.

\section{Shape optimization of artificial leaf}

\subsection{Evaluation functions}

Stiffness, volume and amount of sunlight received were defined as the response functions for evaluating the performance of the leaf.

\subsubsection{Amount of sunlight received}

Figure 10 shows the notations for the amount of sunlight received. The sunlight vector $\vec{L}$ is defined using spherical coordinates as

$$
\vec{L}=(r \sin \theta \cos \varphi, r \sin \theta \sin \varphi, r \cos \theta),
$$

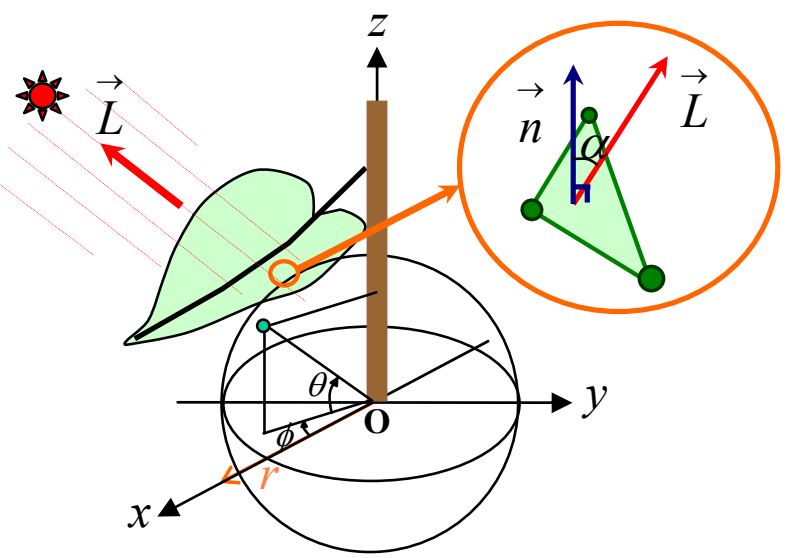

Figure 10: Definition of sunlight vector $L$. 
where $\theta$ and $\varphi$ are the polar and the azimuth angle, respectively, $r$ is the radius, $\vec{n}$ is a unit normal vector to the top surface of the leaf, and $\alpha$ is the angle between $\vec{n}$ and $\vec{L}$. The total amount of sunlight received $Q$ on the top surface of the leaf can then be evaluated using the following equation. It is assumed that only direct light falling on the top surface is considered, and scattered light is ignored, for the sake of simplicity.

$$
Q=\int_{A} \cos \alpha d A,
$$

where $A$ is the blade area.

\subsubsection{Stiffness}

Stiffness is an important property for maintaining the form of leaves against the effects of gravity and winds. In the shape optimization method mentioned above, the compliance which is the inverse of stiffness is actually used. The compliance $C$ is defined as follows:

$$
C=\int_{A} u P d A,
$$

where $u$ and $P$ are the downward displacement and the distributed self-weight force per unit area, respectively. The compliance is evaluated by using the finite element model and the boundary condition shown in Fig. 11. A blade having a maximum length of $85 \mathrm{~mm}$, a maximum width $48 \mathrm{~mm}$, and a constant thickness of $0.2 \mathrm{~mm}$ is discretized by using shell elements. The main vein and the petiole are discretized by bar elements, but the lateral veins are not modelled in this model because an evergreen leaf with a thick blade is considered here. The edge of the petiole is clamped and the downward distributed forces for self-weight are applied to the blade.

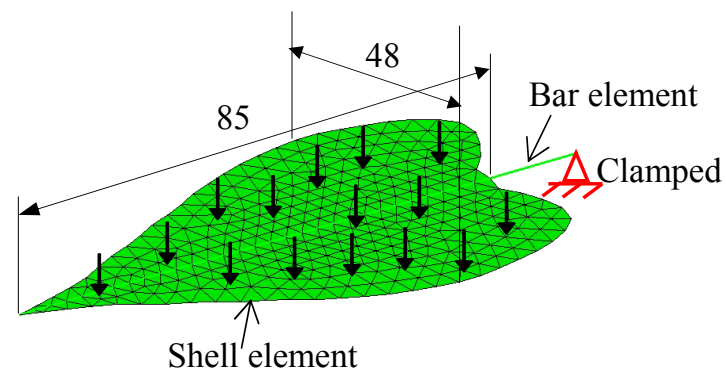

Figure 11: Finite element model and boundary condition for leaf shape optimization.

\subsubsection{Volume}

Assuming that the blade thickness and the cross-sectional area of the main vein are constant, the total volume of the leaf is defined as follows:

$$
V=\int_{A} d A+\int_{l} d l,
$$

where $l$ is the total length of the main vein and the petiole. 


\section{Calculated results}

The pyramid-shaped basis vectors $\boldsymbol{T}_{i}$ in Eq. (4) are defined for all the nodes of the blade (shell elements) and the main vein and the petiole (bar elements) in the normal direction to the initial blade surface in order to obtain or design the optimal free-form of the leaf. The shape optimization method with node-based pyramid-shaped basis vectors is applied to leaf optimization problems involving different combinations of objective and constraint conditions as explained below, and the design variables $x_{i}$ in Eq. (4) are determined. The pyramid-shaped basis vectors are fixed through the optimization process.

\subsection{Maximization of stiffness (problem 1)}

First, a stiffness design problem was solved. The compliance defined by Eq. (8) was used as the objective function. A volume constraint was applied and set as 1.05 times the initial value. Side constraints were set as $-35 \leq x_{i} \leq 35$ on the blade, and $-10 \leq x_{i} \leq 10$ on the main vein, and the all initial values were set as zero. Figure 12 shows the shape convergence histories from the initial shape (a) to the final shape (f). The initial flat shape is changed to the final shape with a Vshaped cross-section for efficiently stiffening the blade, as was expected. Iteration convergence histories of the compliance and the volume are shown in Fig. 13. The values were normalized to those of the initial shape. The

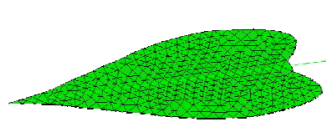

(a) Initial

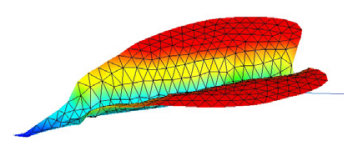

(d) Iteration 20

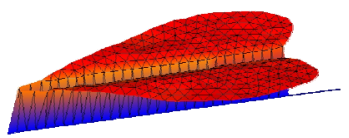

(b) Iteration 5

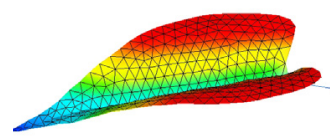

(e) Iteration 30

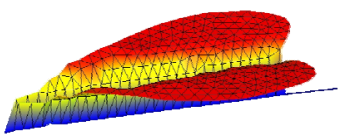

(c) Iteration 10

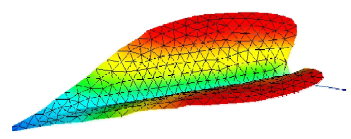

(f) Final

Figure 12: $\quad$ Shape histories of leaf shape optimization (problem 1).

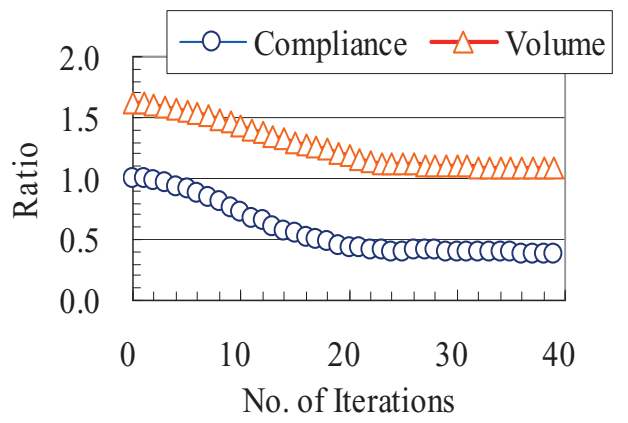

Figure 13: Iteration histories of leaf shape optimization (problem 1). 
compliance was reduced by approximately $62 \%$ while satisfying the given volume constraint. A frontal view of the optimal shape obtained is shown in Fig. 14, where the opening angle gradually shrinks from the tip according to the position along the main vein, i.e. $\alpha_{1}>\alpha_{2}>\alpha_{3}$, as was expected.

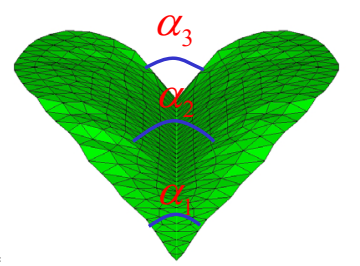

Figure 14: V-shaped angles of final shape.

\subsection{Maximization of amount of sunlight received (problem 2)}

The amount of sunlight received defined by Eq. (7) was maximized. The compliance and the volume constraint were set as less than $35 \%$ and $100 \%$ of the initial shape, respectively. The sunlight vector in Fig. 16 was defined as $\theta=45^{\circ}$ and $\varphi=0^{\circ}$. The other conditions were the same as in problem 1. Figure 16 shows the optimal shape obtained. The result shows that the optimal blade shape with a V-shaped cross-section is tilted toward the sunlight direction to maximize the amount of sunlight received. Iteration convergence histories are shown in Fig. 17. The values were normalized to those of the initial shape. The amount of sunlight received was increased by $23 \%$ over the initial value, while satisfying the given volume and compliance constraints.

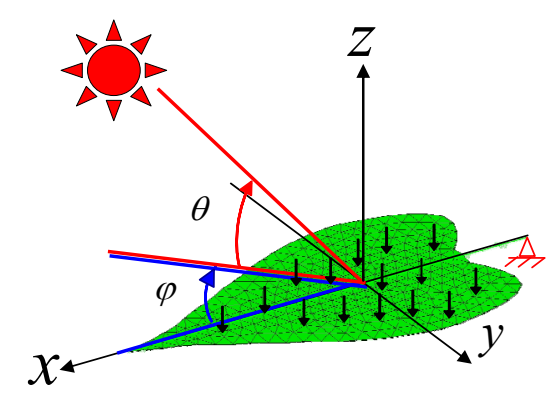

Figure 15: Leaf shape optimization problem $2\left(\varphi=0^{\circ}, \theta=45^{\circ}\right)$. 


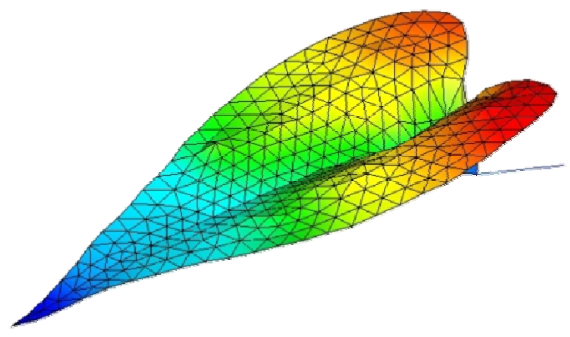

Figure 16: Obtained leaf shape (problem 2).

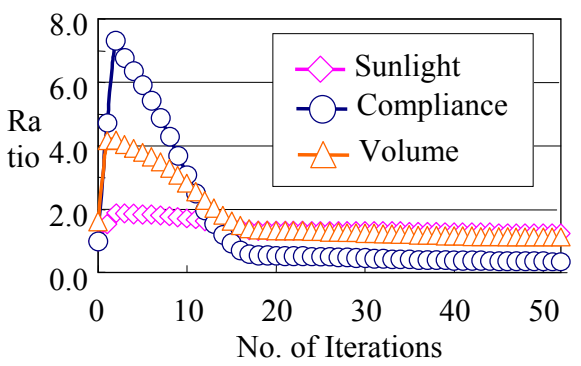

Figure 17: Iteration histories of leaf shape optimization (problem 2).

\subsection{Maximization of stiffness (problem 3)}

The compliance was minimized subject to the sunlight reception constraint. The constraint value was set to the same value as that of the initial shape. The sunlight vector was defined as $\theta=45^{\circ}$ and $\varphi=0^{\circ}$. The other conditions were the same as in problem 1 . The optimal shape obtained and the iteration convergence histories are shown in Figs. 18 and 19, respectively. The result shows that the optimal blade has a different tilt from that in problem 2, and that the V-shaped angle was noticeably enlarged near the petiole to satisfy the trade-off between the amount of sunlight received and stiffness. The compliance was minimized by $63 \%$ of the initial shape, while satisfying the constant sunlight constraint.

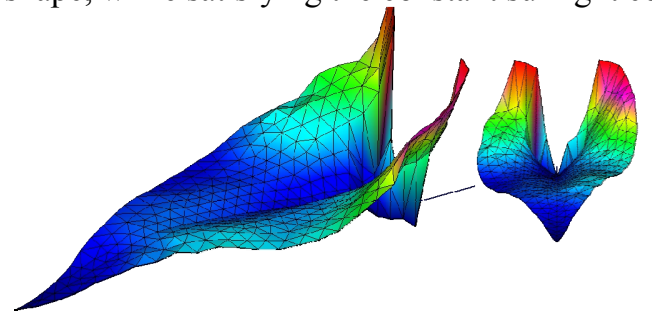

Figure 18: Obtained leaf shape (problem 3). 


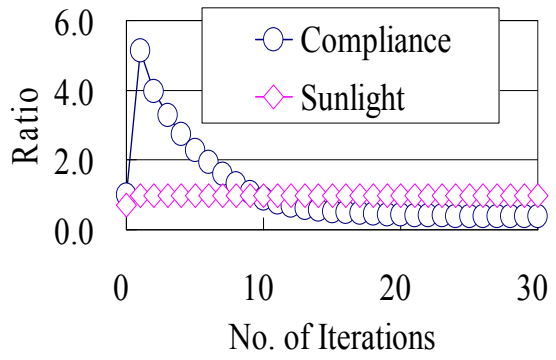

Figure 19: Iteration histories of leaf shape optimization (problem 3).

\section{Conclusion}

A numerical shape optimization method with pyramid-shaped basis vectors was applied to an evergreen leaf model. It was confirmed that the optimal leaf shape was obtained with this method, while satisfying the given constraint conditions. The obtained optimal shape has the following features as was expected: (1) it has a V-shaped cross-section to efficiently stiffen the blade, (2) the V-shaped angle is enlarged along the main vein to the petiole, and (3) the optimal shape is tilted toward the sunlight direction according to the defined polar and azimuth angles in order to receive more sunlight.

\section{References}

[1] S. Vogel, Twist-to-bend ratios of woody structures, $J$. of Experimental Botany, 46, pp.981-985, 1995.

[2] D. Pasini and S. C. Burgess, The structural efficiency of trees, Optimization Mechanics in Nature, M.W. Collins, D. G. Hunt and M. A. Atherton (editors), WIT Press, pp.51-78, 2004.

[3] S. A. Wainwright, W. D. Biggs, J. D. Currey and J. M. Gosline, Mechanical Design in Organisms, Princeton University Press, New Jersey, 1982.

[4] D. F. Cutler, Design in plants, Nature and design, M.W. Collins, M. A. Atherton and J. A. Bryant (editors), WIT Press, pp.95-124, 2005.

[5] D'A. W. Thompson, On Growth and Form, Cambridge University Press, UK, 1917.

[6] C. Mattheck, Design in Nature, Springer, Berlin, 1998.

[7] D. Panini, on the biological shape of the polygonaceae rheum petiole, Int. J. of Design \& Nature and Ecodynamics, 3(1), pp.39-64, 2008.

[8] M. H. Imam, Three-dimensional shape optimization, Int. J. of Num. Meth. Engrg., 18, pp.661-673, 1982.

[9] R. T. Haftka and Z. Gurdal, Elements of Structural Optimization, Kluwer Academic Publishers, Netherlands, 1992. 